\title{
METRIC INEQUALITIES AND CONVEXITY
}

\author{
DOROTHY WOLFE
}

Abstract. Conditions that a given point of a normed linear space is (or is not) a convex combination of $\boldsymbol{n}$ fixed points are given in terms of the metric. The point is said to be metrically dependent if the conditions hold.

The principal aim of this paper is to solve the following problem: Given $q, p_{1}, \cdots, p_{n}$, points in a real normed linear space, what is a necessary and sufficient condition in terms of the metric that $q$ lie inside the convex cover of the other points? The solution is an immediate corollary of Theorem 1, which generalizes a theorem of R. G. Bilyeu.

THEOREM 1. If $q, p_{1}, \cdots, p_{n}$ are members of a real normed linear space $B$ with metric $d$ associated with the norm and if there exist $a_{i}(i=1, \cdots, n)$ such that $a_{i} \geqq 0, \sum_{i=1}^{n} a_{i}=1$, and for each $w$ in the space

then $q=\sum_{i=1}^{n} a_{i} p_{i}$.

$$
d(w, q) \leqq \sum_{i=1}^{n} a_{i} d\left(w, p_{i}\right)
$$

Proof. We shall call $\left\{a_{i}\right\}$ satisfying the conditions of the theorem a set of weights. There is no loss of generality in letting $\sum a_{i} p_{i}=0$. (All summations are from 1 to $n$.) Also, since we look only at the finite dimensional subspace containing the given points, we can consider $B$ finite dimensional.

The proof follows from the fact that for a set of $z$ dense on the boundary of the unit sphere $S$

$$
\lim _{k \rightarrow \infty} \sum a_{i} d\left(k z, p_{i}\right)-d(k z, 0)=0 .
$$

This limit holds for all $z$ such that for each $p_{i}$

$$
\lim _{k \rightarrow \infty}\left[d\left(k z, p_{i}\right)+d\left(k z,-p_{i}\right)-2 d(k z, 0)\right]=0,
$$

since the weighted sum over $i$ of the left side of (2) is (1) plus a similar sum involving $-p_{i}$, both nonnegative.

Received by the editors January 25, 1973.

AMS (MOS) subject classifications (1970). Primary 52A05, 52A50; Secondary 46B99.

Key words and phrases. Normed linear space, metric space, convex combinations, extreme points.

(c) American Mathematical Society 1973 
The limit (2) is obviously equivalent to the existence of the Gateaux differential,

$$
\lim _{h \rightarrow 0} \frac{d(z, h p)-d(z, 0)}{h},
$$

a limit which exists wherever $S$ is smooth (that is, wherever $z$ lies on only one support hyperplane). $S$ is smooth, except possibly at a countable set of points on its boundary [3, pp. 132-134].

To prove the theorem, given any $q \neq 0$, we must produce $w$ such that $d(w, q)>\sum a_{i} d\left(w, p_{i}\right)$. Let $d(q, 0)=\varepsilon$. If $S$ is smooth at $-q / \varepsilon$, set $z=-q / \varepsilon$. In any case, there exists some smooth point $z$ on the boundary of $S$ closer to $-q / \varepsilon$ than $1 / 2$. Then for $k$ sufficiently large

and

$$
\sum a_{i} d\left(p_{i}, k z\right)-d(k z, 0)<\varepsilon / 2
$$

$$
\begin{aligned}
\sum a_{i} d\left(p_{i}, k z\right) & <d(k z, 0)+\varepsilon / 2 \\
& =d(k z,-\varepsilon z)-d(-\varepsilon z, 0)+\varepsilon / 2 \\
& \leqq d(k z, q)+d(-\varepsilon z, q)-d(-\varepsilon z, 0)+\varepsilon / 2 \\
& =d(k z, q)+\varepsilon d(z,-q / \varepsilon)-\varepsilon d(z, 0)+\varepsilon / 2 \\
& <d(k z, q) .
\end{aligned}
$$

This $k z$ is the $w$ we needed.

If $q=\sum a_{i} p_{i}$ for a set of weights $\left\{a_{i}\right\}$, we say that $q$ is convexly dependent on $\left\{p_{1}, \cdots, p_{n}\right\}$. We may add another definition: $q$ is metrically dependent on $\left\{p_{1}, \cdots, p_{n}\right\}$ if there exists a set of weights $\left\{a_{i}\right\}$ such that for all $w$ in the space, $d(w, q) \leqq \sum a_{i} d\left(w, p_{i}\right)$.

Then, since the converse of Theorem 1 is elementary, we can state as a corollary: In a normed linear space, $q$ is convexly dependent on $\left\{p_{1}, \cdots, p_{n}\right\}$ if and only if it is metrically dependent on the same set.

If there exists a set of weights such that the weighted average of distances from a given point $q$ to $n$ fixed points is greater than the average with the same weights from every one of the fixed points, then $q$ is not convexly dependent on the $n$ points. This is the idea behind the following two theorems, which use only the metric structure, not the linearity of the space.

THEOREM 2. Given $n+1$ points $q, p_{1}, \cdots, p_{n}$ in a metric space, if there exists a set of weights $\left\{b_{i}\right\}$ such that

$$
\sum_{i} b_{i} d\left(q, p_{i}\right)>\sum_{i} b_{i} d\left(p_{i}, p_{j}\right) \text { for each } j=1, \cdots, n,
$$

then $q$ is not metrically dependent on $\left\{p_{1}, \cdots, p_{n}\right\}$. 
Proof. Given any set of weights $\left\{b_{i}\right\}$, let $k$ be the value of $j$ for which $\sum_{i} b_{i} d\left(p_{i}, p_{j}\right)$ is a maximum over $j=1, \cdots, n$. Then if, contrary to the conclusion of the theorem, $q$ is metrically dependent, there exists a set of weights $\left\{a_{j}\right\}$ such that $d(w, q) \leqq \sum_{j} a_{j} d\left(w, p_{j}\right)$ for each $w$ (and, in particular, for $\left.w=p_{i}, 1 \leqq i \leqq n\right)$.

But

$$
\begin{aligned}
\sum_{i} b_{i} d\left(p_{i}, p_{k}\right) & =\sum_{j} a_{j} \sum_{i} b_{i} d\left(p_{i}, p_{k}\right) \\
& \geqq \sum_{j} a_{j} \sum_{i} b_{i} d\left(p_{i}, p_{j}\right) \\
& =\sum_{i} b_{i} \sum_{j} a_{j} d\left(p_{i}, p_{j}\right) \\
& \geqq \sum_{i} b_{i} d\left(p_{i}, q\right)
\end{aligned}
$$

contradicting the hypothesis of the theorem.

A somewhat weaker converse requires a condition stronger than the metric dependence inequality for some arbitrary point of the space. The inequality must hold for at least one of the given points $p_{j}$.

THEOREM 3. In a metric space containing $q, p_{1}, \cdots, p_{n}$, if, for every set of weights $\left\{a_{i}\right\}$,

$$
d\left(p_{j}, q\right)>\sum_{i} a_{i} d\left(p_{j}, p_{i}\right) \text { for some } p_{j}
$$

then there exists a set of weights $\left\{b_{i}\right\}$ such that

$$
\sum_{i} b_{i} d\left(q, p_{i}\right)>\sum_{i} b_{i} d\left(p_{i}, p_{j}\right) \text { for each } j .
$$

Proof. Consider the system of $n$ linear inequalities in $x_{1}, \cdots, x_{n}$

(3) $x_{1} d\left(p_{1}, p_{2}\right)+\cdots$

$$
\begin{aligned}
x_{2} d\left(p_{2}, p_{1}\right)+\cdots & +x_{n} d\left(p_{n}, p_{1}\right) & \geqq d\left(q, p_{1}\right), \\
x_{1} d\left(p_{1}, p_{2}\right)+\cdots & +x_{n} d\left(p_{n}, p_{2}\right) & \geqq d\left(q, p_{2}\right), \\
x_{1} d\left(p_{1}, p_{n}\right)+\cdots+ & +x_{n-1} d\left(p_{n-1}, p_{n}\right) & \geqq d\left(q, p_{n}\right) .
\end{aligned}
$$

It is a standard problem in linear programming to find a nonnegative set $\left\{x_{i}\right\}$ such that $\sum_{i} x_{i}$ is a minimum. By the duality theorem [2, p. 73] this set exists if and only if there exists a nonnegative set $\left\{y_{i}\right\}$ such that

$$
\begin{aligned}
& y_{2} d\left(p_{1}, p_{2}\right)+\cdots \quad+y_{n} d\left(p_{1}, p_{n}\right) \leqq 1 \\
& y_{1} d\left(p_{2}, p_{1}\right)+\cdots \quad+y_{n} d\left(p_{2}, p_{n}\right) \leqq 1 \\
& y_{1} d\left(p_{n}, p_{1}\right)+\cdots \quad+y_{n-1} d\left(p_{n}, p_{n-1}\right) \quad \leqq 1 .
\end{aligned}
$$

Furthermore, $\min \left[x_{1}+\cdots+x_{n}\right]=\max \left[y_{1} d\left(q, p_{1}\right)+\cdots+y_{n} d\left(q, p_{n}\right)\right]$. 
Since for every set of weights (i.e., $x_{i}=a_{i}, \sum a_{i}=1$ ), for at least one $p_{j}$ the inequality (3) is not satisfied, the minimum of $\sum x_{i}>1$; hence so is $\max \sum y_{i} d\left(q, p_{i}\right)$. Fixing $y_{1}, \cdots, y_{n}$ at the values for which this maximum is reached, for every $k$ between 1 and $n$

$$
y_{1} d\left(p_{k}, p_{1}\right)+\cdots+y_{n} d\left(p_{k}, p_{n}\right) \leqq 1<y_{1} d\left(q, p_{1}\right)+\cdots+y_{n} d\left(q, p_{n}\right) .
$$

We divide the entire inequality by $\sum y_{i}$. Thus we get a set of weights $b_{j}=y_{j} / \sum y_{i}$ and for each fixed $k$

$$
b_{1} d\left(p_{k}, p_{1}\right)+\cdots+b_{n} d\left(p_{k}, p_{n}\right)<b_{1} d\left(q, p_{1}\right)+\cdots+b_{n} d\left(q, p_{n}\right) .
$$

\section{REFERENCES}

1. R. G. Bilyeu, Metric definition of the linear structure, Proc. Amer. Math. Soc. 25 (1970), 205-206. MR 41 \#4200.

2. Marshall Hall, Jr., Combinatorial theory, Blaisdell, Waltham, Mass., 1967. MR 37 \#80.

3. Frederick A. Valentine, Convex sets, McGraw-Hill Series in Higher Math., McGraw-Hill, New York, 1964. MR 30 \#503.

Department of Mathematics, Widener College, Chester, Pennsylvania 19013 